\title{
Reduced expression levels of the death-associated protein kinase and E-cadherin are correlated with the development of esophageal squamous cell carcinoma
}

\author{
JIANWEN ZHAI, XIAOGANG YANG, YANLI ZHANG, QINGBIN QI, JIGANG HU and QIAOMEI WANG
}

Department of Thoracic Surgery, Affiliated Hospital of Hebei Engineering University, Handan, Hebei 056002, P.R. China

Received November 23, 2012; Accepted January 11, 2013

DOI: $10.3892 / \mathrm{etm} .2013 .916$

\begin{abstract}
The aim of this study was to investigate the expression levels of the death-associated protein kinase (DAPK) and E-cadherin in esophageal squamous cell carcinoma (ESCC) and their correlation with clinical and pathological factors. Immunohistochemistry [streptavidin-peroxidase (SP) method], in situ hybridization, immunoblot assays and reverse transcription-PCR (RT-PCR) were used to detect the expression of DAPK and E-cadherin in the carcinomas and the adjacent normal tissues of 76 cases of esophageal squamous carcinomas. The immunoblot assay indicated that the expression levels of DAPK and E-cadherin were decreased significantly in the ESCC tissue $(\mathrm{P}<0.05)$ when compared with the adjacent normal tissues. The RT-PCR results showed that the mRNA levels of DAPK and E-cadherin were reduced. The abnormal expression of DAPK was highly correlated with the invasiveness and lymphatic metastasis of the cancer. The abnormal expression of E-cadherin was highly correlated with the differentiation and lymphatic metastasis of the cancer. The decreased expression levels of DAPK and E-cadherin correlated with the development of ESCC. The combined detection of DAPK and E-cadherin proteins may be correlated with the degree of malignancy and metastatic potency of ESCC.
\end{abstract}

\section{Introduction}

Death-associated protein kinase (DAPK) (1) is a pro-apoptotic protein identified by scanning apoptosis-initiating genes and tumor-inhibiting genes in the genome. It has been shown that DAPK participates in apoptotic signal transduction pathways, resulting in the suppression of tumor occurrence. Loss of the dynamic balance between cell proliferation and apoptosis

Correspondence to: Dr Jianwen Zhai, Department of Thoracic Surgery, Affiliated Hospital of Hebei Engineering University, No. 81 Congtai Road, Handan, Hebei 056002, P.R. China

E-mail: jwzhai89@yahoo.cn

Key words: death-associated protein kinase, E-cadherin, esophageal squamous cell carcinoma often leads to the accumulation of gene mutations and the occurrence of tumors (2-4).DAPK is located in the 9q34.1 site of the human chromosome and has a molecular mass of $160 \mathrm{kDa}(5)$. It is a serine/threonine protein kinase regulated by calcium/calmodulin and participates in cell survival, apoptosis and tumor suppression (6,7). DAPK expression is absent from a variety of tumor cells and tissues, and the loss of expression is closely correlated with $\mathrm{CpG}$ methylation. DAPK consists of 7 domains, including a core kinase domain, calcium/calmodulin-binding domain, ankyrin repeat region, P-ring, cytoskeleton-binding domain, death domain and serine-rich tail. The core kinase domain is located between the calcium/calmodulin-binding domain and the $\mathrm{N}$-terminus. It is composed of an 11 serine/threonine structure with a conserved lysine residue, which is associated with ATP binding. Mutation of the residue eradicates the apoptotic effect of DAPK (8). Due to its apoptosis promoting and cell adhesion and cell migration inhibiting effects, DAPK plays an important role in tumor development and metastasis $(9,10)$.

E-cadherin is a calcium-dependent glycoprotein that is widely distributed in epithelial cells. It mediates homogeneous cell adhesion, and maintains the integrity of the cell structure and epithelial polarity. Reduced E-cadherin-mediated cell adhesion is a significant cause of tumor invasion and metastasis. The reduction or lack of E-cadherin expression leads to reduced mutual adhesion between the cells. Therefore infiltrative growth and metastasis occur (11). It has been found that ezrin and E-cadherin are involved in the process of esophageal cancer invasion and metastasis (12).

In the current study, in situ hybridization and immunohistochemistry methods were used to detect the expression of DAPK and E-cadherin in the surgically excised esophageal squamous cell carcinoma (ESCC) tissue and the adjacent normal tissues of patients. The study also aimed to further explore the correlation between the expression levels of DAPK and E-cadherin and the development of esophageal cancer in order to identify new molecular markers of esophageal cancers. The immunoblot assay indicated that the expression levels of DAPK and E-cadherin were decreased significantly in the ESCC tissue when compared with the adjacent normal tissues. The reverse transcription (RT)-PCR results showed that the mRNA levels of DAPK and E-cadherin were also reduced. 


\section{Materials and methods}

Clinical data and specimens. Surgical specimens from 76 adult cases of esophageal cancer, who were admitted to the Department of Thoracic Surgery, Affiliated Hospital of Hebei Engineering University between July 2008 and July 2011, were selected and made into a tissue microarray. There were 49 males and 27 females, corresponding to a male to female ratio of $\sim 2: 1$. The patients were aged between 39 and 73 years old with average age of $61 \pm 5.1$ years. The patients did not receive preoperative radiotherapy, chemotherapy or immunotherapy.

The pathological diagnoses were well-differentiated squamous cell carcinoma (26 cases), undifferentiated carcinoma (33 cases) and poorly differentiated carcinoma (17 cases). There were 12 cases of T-I, 21 cases of T-II, 29 cases of T-III and 14 cases of T-IV. There were 35 cases with lymph node metastasis and 41 cases without. All samples were fixed with $40 \mathrm{~g} / 1$ poly formalin and dehydrated conventionally. After embedding in paraffin, the samples were serially sectioned to a thickness of 4-6 $\mu \mathrm{m}$ and stained by H\&E, immunohistochemical and in situ hybridization staining.

Immunohistochemical and in situ hybridization staining. Rabbit anti-human polyclonal antibody to DAPK was purchased from Wuhan Boster Biological Technology, Ltd. (Wuhan, China). The SP immunohistochemistry kit was purchased from Beijing Zhongshan Golden Bridge Biotechnology Co., Ltd. (Beijing, China). The SP method was performed using a 1:150 dilution of the DAPK monoclonal antibody. The tissues were stained with DAB and counterstained with hematoxylin. The staining procedure was carried out strictly in accordance with the manufacturer's instructions. PBS was used as the negative control, instead of primary antibody. The in situ pre-hybridization solution was purchased from Wuhan Boster Biological Technology, Ltd. The 5'-end bio-labeling of the complete phosphorothioate probe were performed by Beijing AudioCodes Biotechnology Co., Ltd. (Beijing, China). The DAPK probe sequence was CAGCTCGCCACCTGCAACGA. The specimens were dewaxed with fresh xylene and dehydrated through an alcohol gradient. Endogenous peroxidase was inactivated for $30 \mathrm{~min}$ by treatment with freshly prepared $0.5 \% \mathrm{H}_{2} \mathrm{O}_{2}$ at room temperature. The DNA-binding protein in the specimens was digested with $3 \%$ fresh citric acid protease $(0.01 \mathrm{~g} / \mathrm{l})$ for $10 \mathrm{~min}$ at $37^{\circ} \mathrm{C}$. Pre-hybridization solution without probe $(20 \mu \mathrm{l})$ was added to each slide which was then maintained at $42^{\circ} \mathrm{C}$ for $4 \mathrm{~h}$. Hybridization solution with probe $(1 \mathrm{ng} / \mathrm{l})$ was hybridized in a moist chamber at $42^{\circ} \mathrm{C}$ for $12 \mathrm{~h}$. The samples were washed with $0.1 \mathrm{X}$ standard sodium citrate (SSC) at $42^{\circ} \mathrm{C}$ and SA-Bio-AP was added. After incubating at $37^{\circ} \mathrm{C}$ for $10 \mathrm{~min}$ and then rinsing, BCIP/NBT were added and the samples were kept in the dark for 2-4 $\mathrm{h}$ to allow the color reaction to occur. A sample without the probe was the negative control.

Positive criteria of immunohistochemistry and in situ hybridization staining. Positively stained DAPK protein is observed as pale yellow or brown granules in the cytoplasm. Five fields in the high magnification image were randomly selected and $\geq 200$ cells were observed in each field. The positive cell percentage and color depth were determined (13). A nine-point
Table I. Primers used for RT-PCR.

\begin{tabular}{lcl}
\hline Gene & F/R & Primer sequence \\
\hline GAPDH & F & 5'-GTGGGGCGCCCCAGGCACCA-3' \\
GAPDH & R & 5'-CTCCTTAATGTCACGCACGATTT-3' \\
DAPK & F & 5'-AGCATATCTACTAGATATCTGAT-3' \\
DAPK & $\mathrm{R}$ & 5'-GGTACTCCACGTCGACGAGAC-3' \\
E-cadherin & F & 5'-CAGTGAGCG GAGATAGTGCC-3' \\
E-cadherin & R & 5'-CAAAAGTTGGAAAGCCCGTG-3' \\
\hline
\end{tabular}

RT-PCR, reverse transcription-PCR; DAPK, death-associated protein kinase; F, forward; R, reverse.

scoring system was adopted. The positive cell proportion was determined and scored as follows: 1 point for $<10 \%$ positive cells, 2 points for $10-50 \%$ and 3 points for $>50 \%$. The color depth was also scored: 0 point for negative staining, 1 point for slight yellow color staining, 2 points for moderate yellow color staining and 3 points for pale-brown color staining. Scores of $0-1,2$ and $\geq 3$ are represented by $(-),(+)$ and $(++)$, respectively, with (++) indicating normal expression and (-) and (+) indicating missing or weak expression.

The positive expression of E-cadherin is shown as yellow or brown-yellow stained fine particles in the cell membrane, while negative expression is shown as expression in the cytoplasm but not in the membrane. According to the criteria of Bajbouj (14), (-) signifies negative staining, (+) signifies a positive cell proportion of $<75 \%$ and $(++)$ signifies a positive cell proportion of $\geq 75 \%$; (++) indicates normal expression, while $(-)$ and (+) indicate missing or weak expression.

Quantitative RT-PCR. The esophageal cancer tissues and the adjacent normal mucosa from the surgical specimens of 76 cases were isolated using the RNeasy FFPE kit (cat. \#73504, Qiagen, Valencia, CA, USA) and stored at $-80^{\circ} \mathrm{C}$ for further analyses. Quantitative RT-PCR analysis of DAPK and E-cadherin mRNA levels in the tissues were performed. The RT-PCR experiments were repeated at least 3 times. RNA was reverse transcribed into cDNA using random primers in a Reverse Transcription II system (Promega, Madison, WI, USA) according to the manufacturer's instructions. Expression of DAPK and E-cadherin mRNAs was quantified by quantitative PCR using an ABI Prism Sequence Detection system (Applied Biosystems, Carlsbad, CA, USA). The primers used in this study are listed in Table I. An assay reagent containing premixed primers and a VIC-labeled probe (Applied Biosystems; cat. \#4310884E) was used to quantify the expression of endogenous GAPDH mRNA. Template-negative and RT-negative conditions were used as controls. Amplification of the endogenous GAPDH cDNA was monitored. The levels (mean values) of DAPK and E-cadherin transcripts in the patients were calculated.

Immunoblot assays. Total proteins were harvested from the esophageal cancer tissues and the adjacent normal mucosa from the surgical specimens of 76 cases. The proteins were separated by $10 \%$ SDS/PAGE and then subjected to immunoblot analyses. The primary antibodies against 
Table II. Expression of DAPK and E-cadherin in esophagus squamous carcinomatissue and adjacent normal tissue (\%).

\begin{tabular}{lcccccr}
\hline Category & DAPK(+) & DAPK(-) & P-value & E-cadherin(+) & E-cadherin(-) & P-value \\
\hline Normal tissue & $65(85.5)$ & $11(14.5)$ & & $74(97.36)$ & $2(2.63)$ & \\
Cancer tissue & $27(35.5)$ & $49(64.5)$ & $<0.001^{\text {a }}$ & $21(27.63)$ & $55(72.36)$ & $<0.001^{\text {b }}$ \\
\hline
\end{tabular}

Percentage of positive staining. ${ }^{\mathrm{a}} \chi^{2}=39.762,{ }^{\mathrm{b}} \chi^{2}=78.849$. DAPK, death-associated protein kinase.

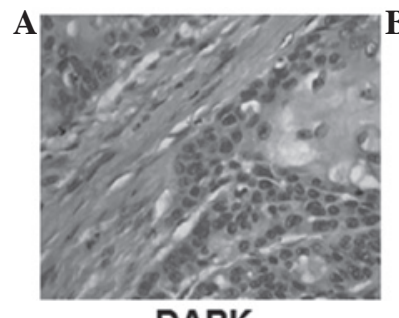

DAPK

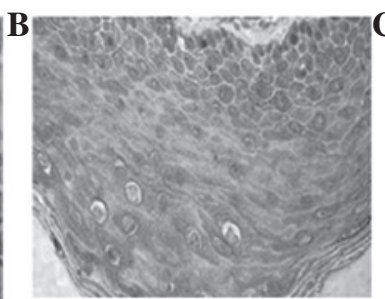

DAPK

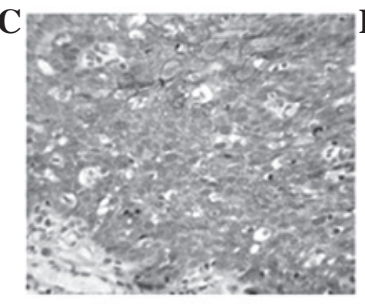

E-cadherin

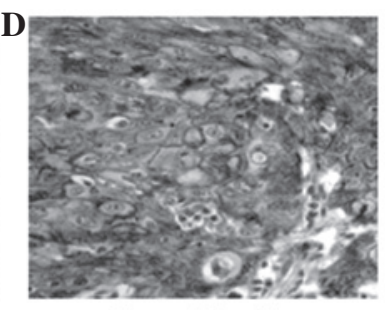

E-cadherin

Figure 1. Immunohistochemical staining of DAPK and E-cadherin in esophagus squamous carcinoma and adjacent normal tissues. Cells with positive expression of target protein were stained. (A) Expression of DAPK in stratum spinosum and stratum corneum of adjacent normal tissues; (B) Expression of DAPK in the cytoplasm of cancer cells; (C) Expression of E-cadherin in the stratum spinosum and horny layer of adjacent normal tissues; (D) Expression of E-cadherin in the epicytes of cancer cells (all SP x400). DAPK, death-associated protein kinase.

DAPK ( 160 kDa), E-cadherin ( 120 kDa) and $\beta$-actin were purchased from Santa Cruz Biotechnology, Inc. (anti-DAPK, cat. \#sc-10805, 1:200; anti-E-cadherin, cat. \#sc-7870, 1:200; anti- $\beta$-actin, cat. \#sc-130301, 1:10,000; Santa Cruz, CA, USA). The secondary antibodies used in this study were goat anti-mouse IgG-HRP (cat. \#sc-2005, 1:10,000; Santa Cruz Biotechnology, Inc.) and anti-rabbit IgG-HRP (cat. \#31460, 1:5,000; Pierce Biotechnology, Rockford, IL, USA). Bound antibodies were detected using an ECL system (Pierce Biotechnology). The immunoblot experiments were repeated at least 3 times. The mean normalized optical density (OD) of DAPK and E-cadherin protein bands relative to the OD of the $\beta$-actin band from the same individual was calculated.

Statistical analysis. All statistical calculations were performed using SPSS 13.0 statistical software. Continuous variables were summarized as the mean values (mean \pm standard error) and compared using the independent sample t-test. $\mathrm{P}<0.05$ was considered to indicate a statistically significant result. In addition, the $\chi^{2}$ test and Spearman's rank correlation coefficient analysis were used. $\alpha=0.05$ was considered to indicate a statistically significant difference.

\section{Results}

Expression of DAPK and E-cadherin in ESCC and adjacent normal mucosa. DAPK protein is localized in the cytoplasm and presents as pale yellow or brown granules when stained. No positive staining was observed in the control tissue (Fig. 1A and B). The positive expression of E-cadherin is mainly localized in the cell membrane, and presents as brownish-yellow granules when stained.

E-cadherin expression was observed in the epithelial cells of the esophageal carcinoma cancer tissue and normal tissue and was strongly expressed in the basal cells and spinous cell layers
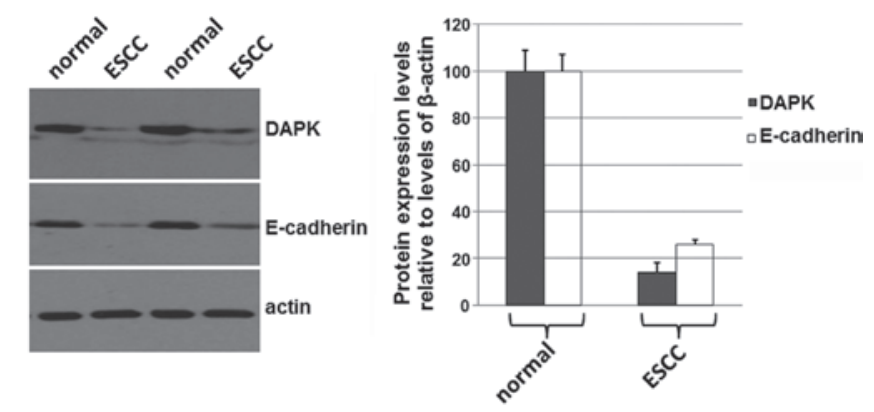

Figure 2. Immunoblots of DAPK and E-cadherin expression in the ESCC tissues and the adjacent normal tissues of patients. The total proteins were harvested from the ESCC and the adjacent normal tissues, separated by $10 \%$ SDS/PAGE and then subjected to immunoblot analyses. Bound antibodies were detected using an ECL system (Pierce Biotechnology). The experiments were repeated at least three times. (A) Representative blots. (B) The mean normalized optical density (OD) of DAPK and E-cadherin protein bands relative to the OD of $\beta$-actin bands was calculated. ESCC, esophageal squamous cell carcinoma; DAPK, death-associated protein kinase.

in the squamous epithelial tissues of the normal esophagus (Fig. 1C and D). There were significantly differences in DAPK and E-cadherin expression between the esophageal cancer tissue and the adjacent normal epithelium tissue, as shown in Table II.

Correlation between expression of DAPK and E-cadherin and clinical and biological behavior of ESCC. No correlations were noted between DAPK expression and the age, gender and tumor differentiation degree of the patients. However, there were correlations between DAPK expression and the tumor invasion depth and lymph node metastasis.

No correlations were observed between E-cadherin expression and the age, gender and tumor invasion depth of the patients. There were significant correlations between E-cadherin expression and tumor differentiation and lymph node metastasis, as shown in Table III. 
Table III. Correlation between expression of DAPK and E-cadherin and clinical parameters of esophageal squamous carcinoma.

\begin{tabular}{|c|c|c|c|c|c|c|c|c|c|c|c|}
\hline \multirow{2}{*}{$\begin{array}{l}\text { Clinical and } \\
\text { pathological factors }\end{array}$} & \multirow[b]{2}{*}{$\mathrm{n}$} & \multicolumn{4}{|c|}{ DAPK expression } & \multirow[b]{2}{*}{ P-value } & \multicolumn{4}{|c|}{ E-cadherin expression } & \multirow[b]{2}{*}{ P-value } \\
\hline & & - & + & ++ & $\%$ & & - & + & ++ & $\%$ & \\
\hline \multicolumn{12}{|l|}{ Gender } \\
\hline Male & 49 & 21 & 10 & 18 & 36.7 & & 25 & 10 & 14 & 28.5 & \\
\hline Female & 27 & 11 & 7 & 9 & 33.3 & & 17 & 3 & 7 & 25.9 & \\
\hline $\mathrm{Z}_{\mathrm{c}}$ & & & -0.205 & & & 0.838 & & -0.383 & & & 0.452 \\
\hline \multicolumn{12}{|l|}{ Age (years) } \\
\hline$<60$ & 44 & 17 & 11 & 16 & 36.3 & & 27 & 4 & 13 & 29.5 & \\
\hline$\geq 60$ & 32 & 16 & 5 & 11 & 34.3 & & 15 & 9 & 8 & 25.0 & \\
\hline $\mathrm{Z}_{\mathrm{c}}$ & & & -0.196 & & & 0.844 & & -0.698 & & & 0.485 \\
\hline \multicolumn{12}{|l|}{ Tumor size } \\
\hline$<5 \mathrm{~cm}$ & 33 & 14 & 9 & 10 & 30.3 & & 16 & 8 & 9 & 27.2 & \\
\hline$\geq 5 \mathrm{~cm}$ & 43 & 18 & 8 & 17 & 39.5 & & 26 & 5 & 12 & 27.9 & \\
\hline $\mathrm{Z}_{\mathrm{c}}$ & & & -0.475 & & & 0.635 & & -0.695 & & & 0.487 \\
\hline \multicolumn{12}{|l|}{ Degree of differentiation } \\
\hline High & 26 & 11 & 6 & 9 & 34.6 & & 8 & 6 & 12 & 46.2 & \\
\hline Moderate & 33 & 14 & 7 & 12 & 36.3 & & 21 & 5 & 7 & 21.2 & \\
\hline Low & 17 & 7 & 4 & 6 & 35.2 & & 13 & 2 & 2 & 11.7 & \\
\hline$\chi^{2}$ value & & & 0.006 & & & 0.997 & & 10.148 & & & 0.006 \\
\hline \multicolumn{12}{|l|}{ Infiltration degree } \\
\hline $\begin{array}{l}\text { Did not infiltrate } \\
\text { outer membrane }\end{array}$ & 31 & 22 & 4 & 5 & 16.1 & & 16 & 5 & 10 & 32.2 & \\
\hline Infiltrated outer & & & & & & & & & & & \\
\hline membrane and tissue & 45 & 10 & 13 & 22 & 48.9 & & 26 & 8 & 11 & 24.4 & \\
\hline $\mathrm{Z}_{\mathrm{c}}$ & & & -3.981 & & & 0.000 & & -0.775 & & & 0.450 \\
\hline \multicolumn{12}{|l|}{ Lymph node metastasis } \\
\hline Yes & 41 & 13 & 8 & 20 & 48.7 & & 28 & 7 & 6 & 14.6 & \\
\hline No & 35 & 19 & 9 & 7 & 20.0 & & 14 & 6 & 15 & 42.8 & \\
\hline $\mathrm{Z}_{\mathrm{c}}$ & & & -2.409 & & & 0.016 & & -2.758 & & & 0.006 \\
\hline
\end{tabular}

DAPK, death-associated protein kinase.

Expression levels of DAPK and E-cadherin are decreased in ESCC tissues. To determine if the expression levels of DAPK and E-cadherin were correlated with the development of carcinomas, the total proteins were isolated from esophageal cancer tissues and the adjacent normal mucosa from surgery specimens of the 76 cases. Western blotting was performed. As shown in Fig. 2A, the expression levels of DAPK and E-cadherin were decreased in ESCC compared with the levels in the adjacent normal tissues. The levels of $\beta$-actin were used as a loading control. The mean normalized OD of DAPK and E-cadherin protein bands relative to the OD of $\beta$-actin bands from each of patients was calculated (Fig. 2B). The results shown in Fig. 2 suggest that expression levels of DAPK and E-cadherin are decreased in ESCC tissues, which may be related to the development of ESCC.

Levels of DAPK and E-cadherin $M R N A$ are decreased in ESCC tissues. To determine if levels of DAPK and E-cadherin mRNAs were decreased in ESCC tissues, real-time PCR was performed. As shown in Fig. 3, the expression levels of

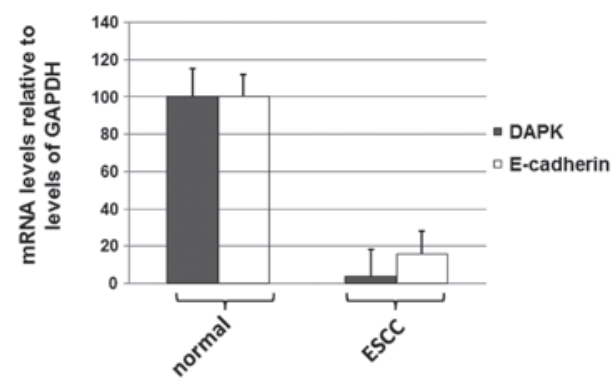

Figure 3. Quantitative RT-PCR analysis of DAPK and E-cadherin mRNA levels in ESCC and adjacent normal tissues. Total RNA was harvested from tissues using the RNeasy FFPE kit (Qiagen) according to the manufacturer's instructions. The RT-PCR experiments were repeated at least 3 times. RNA was reverse transcribed into cDNA using random primers in a Reverse Transcription II system (Promega) according to the manufacturer's instructions. Expression of DAPK and E-cadherin mRNAs was quantified by quantitative PCR using an ABI Prism Sequence Detection System (Applied Biosystems). Template-negative and RT-negative conditions were used as controls. Amplification of the endogenous GAPDH cDNA was monitored. Levels (mean values) of DAPK and E-cadherin transcripts in patients were calculated. RT-PCR, reverse transcription-PCR; ESCC, esophageal squamous cell carcinoma; DAPK, death-associated protein kinase. 
DAPK and E-cadherin mRNA were reduced in ESCC tissues compared with the levels in the adjacent normal tissues. The results shown in Fig. 3 suggest that levels of DAPK and E-cadherin mRNA were decreased in ESCC tissues.

\section{Discussion}

In the current study, it is shown that the DAPK gene is expressed in esophageal cancer tissue and adjacent normal esophageal tissues. However, the expression levels of these proteins are significantly reduced in cancer tissues, as revealed by the results of the immunoblot assay and RT-PCR. The results also indicate that the abnormal DAPK gene expression is closely correlated with the invasion depth of esophageal squamous cell carcinoma and lymph node metastasis, which suggests that the abnormal expression of DAPK may be an indicator of prognosis. E-cadherin expression is associated with the degree of esophageal cancer differentiation and lymph node metastases, but there is no significant correlation with age, gender, tumor length or the depth of invasion. It was also identified in this study that the E-cadherin expression rate in cancer tissues with lymph node metastasis was significantly lower than that in cancer tissues without lymph node metastasis $(\mathrm{P}<0.05)$. It was also observed that the E-cadherin expression rate in poorly differentiated cancer tissue was significantly lower than that in well-differentiated cancer tissues $(\mathrm{P}<0.05)$, indicating that the reduction of E-cadherin expression is associated with the differentiation of esophageal squamous cell carcinoma and lymph node metastasis. However, no significant difference between the E-cadherin expression with or without fiber membrane infiltration was identified, suggesting that E-cadherin is not correlated with the invasion depth of esophageal squamous cell carcinoma. Further analysis of in situ hybridization and immunohistochemical detection showed consistent DAPK and E-cadherin positive expression in esophageal carcinoma. The DAPK protein expression rate and E-cadherin expression were positively correlated $(\mathrm{P}<0.01)$, suggesting the correlation of DAPK and E-cadherin in tumor invasion and metastasis.

The invasion and metastasis of tumor cells are processes involving multiple stages, steps and factors. They are associated with the main characteristics of the tumor cells, overall host immune status and the characteristics of transformed local tissues. DAPK and E-cadherin expression correlate with esophageal squamous cell carcinoma and metastasis, suggesting that DAPK and E-cadherin may have a synergistic effect. In situ hybridization and immunohistochemical detection were used to analyze the expression of DAPK and E-cadherin in esophageal squamous cell carcinoma, which initially showed the reduction or lack of DAPK expression may lead to the development of esophageal cancer. The combined methods contribute to the comprehensive diagnosis of ESCC, the degree of malignancy, metastatic potential and prognosis.

\section{Acknowledgements}

This study was supported by the Science and Technology Department of Hebei Province: Hebei Technology Support Program (Grant \#112061176D).

\section{References}

1. Deiss LP, Feinstein E, Berissi H, Cohen O and Kimchi A: Identification of a novel serine/threonine kinase and a novel 15-kD protein as potential mediators ofthe gamma interferon-induced cell death. Genes Dev 9: 15-30, 1995.

2. Brabender J, Arbab D, Huan X, et al: Death-associated protein kinase (DAPK) promoter methylation and response to neoadjuvant radiochemotherapy in esophageal cancer. Ann Surg Oncol 16: 1378-1383, 2009.

3. Tong A, Lynn G, Ngo V, et al: Negative regulation of Caenorhabditis elegans epidermal damage responses by death-associated protein kinase. Proc Natl Acad Sci USA 106: 1457-1461, 2009.

4. Li H, Ray G, Yoo BH,Erdogan M and Rosen KV: Down-regulation of death-associated protein kinase-2 is required for betacatenin-induced anoikis resistance of malignant epithelial cells. Biol Chem 284: 2012-2022, 2009.

5. Zalckvar E, Berissi H, Eisenstein $M$ and Kimchi A: Phosphorylation of Beclin 1 by DAP-kinase promotes autophagy by weakening its interactions with Bcl-2 and Bcl-XL. Autophagy 5: 720-722, 2009.

6. Yanagawa N, Osakabe M,Hayashi M, Tamura G and Motoyama T: Detection of HPV-DNA, p53 alterations, and methylation in penile squamous cell carcinoma in Japanese men. Pathol Int 58: 477-482, 2008

7. Hoffmann AC, Vallböhmer D, Prenzel K, et al: Methylated DAPK and APC promoter DNA detection in peripheral blood is significantly associated with apparent residual tumor and outcome. J Cancer Res Clin Oncol 135: 1231-1237, 2009.

8. Fendri A, Masmoudi A, Khabir A, et al: Inactivation of RASSF1A, RARbeta2 and DAP-kinase by promoter methylation correlates with lymph node metastasis in nasopharyngeal carcinoma. Cancer Biol Ther 8: 444-451, 2009.

9. Santos-García A, Abad-Hernández MM, Fonseca-Sánchez E, et al: E-cadherin, laminin and collagen IV expression in the evolution from dysplasia to oral squamous cell carcinoma. Med Oral Patol Oral Cir Bucal 11: E100-E105, 2006.

10. Zhai JW, Yang XG, Yang FS, Hu JG and Hua WX: Expression and clinical significance of Ezrin and E-cadherin in esophageal squamous cell carcinoma. Chin J Cancer 29: 317-320, 2010.

11. Gao DL, Li SL, Chen KS, Zhao ZH, Zhao QM, Liu ZW and Zhang YH: The inhibition of metalloproteinase in RECK expression in esophageal squamous cell carcinoma and its biological significance. World J Gastroenterol 16: 1634-1638, 2008.

12. Gonzalez MA, Pinder SE, Wencyk PM, et al: An immunohistochemical examination of the expression of E-cadherin, alpha- and beta/gamma-catenins, and alpha2- and betal-integrins in invasive breast cancer. J Pathol 187: 523-529, 1999.

13. Lou C, Yang B, Gao YT, Wang YJ, Nie FH, Yuan Q, Zhang CL and Du Z: Aberrant methylation of multiple genes and its clinical implication in hepatocellularcarcinoma. Zhonghua Zhong Liu Za Zhi 30: 831-836, 2008 (In Chinese).

14. Bajbouj K, Poehlmann A, Kuester D, et al: Identification of phosphorylated p38 as a novel DAPK-interacting partner during TNFalpha-induced apoptosis in colorectal tumor cells. Am J Pathol 175: 557-570, 2009. 